\title{
Mineralization and bone regeneration using a bioactive elastin-like recombinamer membrane
}

\author{
Esther Tejeda-Montes, Alexey Klymov, M. Reza Nejadnik, Matilde Alonso, \\ J.Carlos Rodriguez-Cabello, X. Frank Walboomers, Alvaro Mata
}

\section{Introduction}

Bone grafts are extensively used in bone healing therapies that require significant osteoconductive and osteoinductive enhancement. Despite a number of well-known negative consequences, autogenous cancelous bone continues to be the preferred bone graft option and a major target to replace [1]. Scaffolds based on collagen [2], hyaluronic acid [3], chitosan [4], biological composites [5], and self-assembling materials [6] have been and continue to be investigated as three-dimensional bone graft alternatives.

In an attempt to further enhance bone regeneration therapies, and taking advantage of the benefits of the periosteum, the use of periosteal grafts has emerged as an attractive strategy [7]. An ideal periosteal graft would not only provide a physical structure that facilitates osteoconduction, but also osteoinductive signals that stimulate osteogenesis and ultimately promote biomineralization [8]. Membranes made of amniotic tissue [9], chitosan-silica [10] or silk fibroin nanofibers [11] have been reported to induce osteoblastic differentiation in vitro. Also, a variety of in vitro mineralizing membranes based on chitosan/bioactive glass nanoparticles [12], platelet-rich fibrin functionalized with alkaline phosphatase (ALK) [13], polycaprolactone fibers incorporating nano-apatite particles [14] or forsterite nanopowder [15], and collagen [16] have been developed. In addition, the capacity of membranes to enhance bone regeneration in vivo has been reported. Examples include membranes based on a modified polylactide/polyglycolide acid polymer [17], collagen membranes combined with a porous titanium membrane [18], or chitosan bioelectric membranes capable of accelerating bone fracture healing by electric stimulation [19]. 
However, the success of periosteal grafts has been restricted by limitations associated with the lack of biocompatibility, absence of bioactivity, poor mechanical properties, or early degradation [20].

A possible alternative to overcome these limitations may be found in the use of molecularly designed materials made from peptides and proteins. This approach offers a much higher level of tuneability, spatio-temporal control, bioactivity, and stimulation of bone formation. Towards this goal, phosphorylated serine $[\mathrm{S}(\mathrm{P})][6]$, MLPHHGA heptapeptide [21], the bone sialoprotein sequence E7PRGDT [22], or the statherin-derived protein sequence DDDEEKFLRRIGRFG [23] have been used and reported to promote mineralization in vitro. Elastin-like recombinamers (ELRs), genetically engineered protein-based polymers inspired by the extracellular matrix protein elastin [24], are especially attractive due to their molecular versatility, biomimetic character, biocompatibility, good mechanical properties, and biodegradability [24]. These molecules are mainly composed of the repeating pentapeptide domain VPGXG (where X could be any amino acid apart from proline) and can be designed to contain additional bioactive sites such as RGDS or REDV [25].

In an effort to bioengineer a bioactive membrane for bone regeneration that takes advantage of the potential benefits of these biomolecular sequences, we have recently reported on a couple of strategies based on peptide and protein-based materials [26,27]. In particular, we have described the fabrication and characterization of thin robust ELR membranes comprising the bioactive epitope DDDEEKFLRRIGRFG, and their capacity to promote osteogenesis in vitro [25]. The present work provides further evidence of the potential use of these membranes for bone regeneration applications. First, we describe the in vitro mineralization and osteogenic properties of the bioactive ELR membranes tested under biomimetic conditions in both static and dynamic culture settings. Then, we report on the bone regeneration capacity of these membranes using an orthotopic critical-size rat calvarial defect model.

\section{Materials and methods \\ 2.1. ELR molecules}

Four ELRs molecules were supplied by Technical Proteins NBT S.L. (Valladolid, Spain). The materials consisted of repeating pentapeptide domains of VPGIG and VPGKG to provide structural integrity including the amino acid lysine (K) to serve as a cross-linking point (IK), that incorporated the peptide RGDS for mesenchymal stem cell adhesion (RGDS), the peptide DDDEEKFLRRIGRFG for nucleation of mineralization (HAP) and an ELR that combined the later and the RGDS sequence (HAP-RGDS) (Table 1).

\subsection{Membrane fabrication}

Membranes were fabricated according to a recently reported method [25,27] (Fig. 1aec). The ELR molecules were dissolved in anhydrous dimethylformamide (DMF) (SigmaeAldrich, Taufkirchen, Germany) at room temperature, and then mixed with hexamethyl diisocyanate (HDI) (SigmaeAldrich, Taufkirchen, Germany) (Table 1). Four ELR membranes were fabricated from the different ELR molecules, containing either one of the bioactive sequences (RGDS, HAP, and HAP-RGDS) or without any bioactivity (IK).
2.3. Fabrication and characterization of topographically patterned ELR membranes

In order to fabricate topographically patterned ELR membranes, a patterned PDMS mould was fabricated by soft lithography techniques as previously reported [27]. The features consisted of channels that were $7 \mathrm{~mm}$ high, $10 \mathrm{~mm}$ wide, and separated by $10 \mathrm{~mm}$ wide ridges (Channels) (Fig. 1c). Membrane fabrication and pattern reproducibility were analyzed by qualitative observations using scanning electron microscopy (SEM) and profilometry.

\subsection{Mechanical stimulation}

A custom-made stretching device [28] was used in this study (Fig. 1d). The system applied uniform, cyclic, uniaxial strain to elastic silicone dishes that had $18 \mathrm{~cm}^{2}$ surface growth areas, in which the ELR membranes were immobilized. A cyclic stretching magnitude of $8 \%$ at $1 \mathrm{~Hz}$ frequency with intermittent stretch duration of $15 \mathrm{~min}$ stretch/15 min rest for $16 \mathrm{~h}$, followed by $8 \mathrm{~h}$ of rest was applied. This process was defined as dynamic condition throughout the document. All experiments were performed inside of a cell culture incubator controlling temperature and $\mathrm{CO}_{2}$ level $\left(37{ }^{\circ} \mathrm{C}\right.$ and $\left.5 \% \mathrm{CO}_{2}\right)$. The static condition was used as control. This procedure was applied in Section 2.5 and 2.6.

\subsection{Membrane mineralization in simulated body fluid}

Membranes made of IK, RGDS, HAP and HAP-RGDS ELR molecules, comprising or not microtopographical channels in a rectangular shape $\left(7.5 \times 15 \mathrm{~mm}^{2}\right)$ were analyzed in this study. The experiment was performed applying a mechanical stimulation described in the Section 2.4, while the samples were incubated in $3 \mathrm{ml}$ of simulated body fluid (SBF) which was replaced daily for 7 days. ELR membranes incubated in milli-Q water in static conditions were used as control. SBF was pre pared following a previously reported protocol [29]. The SBF solution was prepared using $50 \mathrm{~mm}$ Tris $\left(\mathrm{CH}_{2} \mathrm{OH}\right)_{3} \mathrm{CNH}_{2}, 136.8 \mathrm{~mm} \mathrm{NaCl}, 3 \mathrm{~mm} \mathrm{KCl}, 1.5 \mathrm{~mm} \mathrm{MgCl} \mathrm{M}_{2} 6 \mathrm{H}_{2} \mathrm{O}$, $2.5 \mathrm{mM} \mathrm{CaCl}_{2} \mathrm{H}_{2} \mathrm{O}, 1 \mathrm{mM} \mathrm{K}_{2} \mathrm{HPO}_{4}, 4.15 \mathrm{mM} \mathrm{NaHCO}_{3}$, and $0.5 \mathrm{mM} \mathrm{Na}_{2} \mathrm{SO}_{4} 10 \mathrm{H}_{2} \mathrm{O}$. In order to prevent the unwanted deposition of calcium phosphate minerals during the storage of SBF, two stock solutions were prepared. The first stock solution was made with the reagents Tris $\left(\mathrm{CH}_{2} \mathrm{OH}\right)_{3} \mathrm{CNH}_{2}, \mathrm{NaCl}, \mathrm{KCl}, \mathrm{K}_{2} \mathrm{HPO}_{4}, \mathrm{NaHCO}_{3}, \mathrm{Na}_{2} \mathrm{SO}_{4} 10 \mathrm{H}_{2} \mathrm{O}$ and the second stock solution with the reagents Tris $\left(\mathrm{CH}_{2} \mathrm{OH}\right)_{3} \mathrm{CNH}_{2}, \mathrm{NaCl}, \mathrm{MgCl}_{2} 6 \mathrm{H} 2 \mathrm{O}$ $\mathrm{CaCl}_{2} \mathrm{H}_{2} \mathrm{O}$. After adjusting the $\mathrm{pH}$ of the stock solutions to 7.4 , they were stored at $4{ }^{\circ} \mathrm{C}$. Prior to the mineralization experiments, the stock solutions were mixed and diluted with milli-Q water to obtain the SBF concentration of the reagents. The resulting SBF solution was filtered using a surfactant-free cellulose acetate filter unit (pore size $0.2 \mathrm{~mm}$ ) to eliminate impurities and used in the mineralization experiments. In order to assess the mineralization potential of the ELR membranes, in vitro mineralization in SBF was investigated using different techniques. Each sample was assayed in triplicate and the experiment was repeated twice.

\subsubsection{Calcium content}

ELR membranes were rinsed twice with milli-Q water after 7 days of incubation in SBF solution. Then, $100 \mathrm{ml}$ of $0.5 \mathrm{M}$ acetic acid was added in each membrane. The samples were maintained under agitation overnight at room temperature. The supernatant was recollected and calcium content was determined using an orthocresolphthalein complexone method [30]. The calcium content was measured on a fluorescence microplate reader (Bio-Tek Instruments Inc., Winooski, VT, USA) at $570 \mathrm{~nm}$, and determined by extrapolating known values from a standard curve. All samples were assayed twice.

\subsubsection{Mineral characterization}

The mineral on Smooth HAP membranes incubated in SBF for 7 days in static condition was characterized by time-of-flight secondary ion mass spectroscopy (TOF-SIMS) and scanning electron microscopy fitted with an energy dispersive spectrometer (SEM-EDS). Smooth HAP membranes incubated in milli-Q water were used as control.

Membranes analyzed by TOF-SIMS were rinsed twice with mili-Q water, frozen at $-80{ }^{\circ} \mathrm{C}$ overnight and freeze-dried for $24 \mathrm{~h}$. The TOF-SIMS (TOF-SIMS IV, ION-TOF, Germany) operated at a pressure of $5 \times 10^{-9}$ mbar. Samples were bombarded with a

Table 1

Sequence, functionality (bioactivity), concentration, and ELR:Cross-linker ratio of the ELR materials

\begin{tabular}{|c|c|c|c|c|}
\hline ELR Material & ELR sequence (bioactive sequence) & Bioactivity & ELR Con.(\%) & ELR: Cross-linker ratio \\
\hline IK & (VPGIG VPGIG VPGKG VPGIG VPGIG) 24 & Control & 4 & $1: 0.75$ \\
\hline RGDS & $\left.\left[\left[(V P G \mid G)_{2}(V P G K G)(V P G \mid G)_{2}\right]_{2} \text { AVTGRGDSPASS[(VPGIG) } 2(V P G K G)(V P G \mid G)_{2}\right]_{2}\right] 6$ & Cell Adhesion & 3.5 & $1: 0.50$ \\
\hline HAP & {$\left[\left[(\mathrm{VPG} \mid \mathrm{G})_{2}(\mathrm{VPGKG})(\mathrm{VPG} \mid \mathrm{G})_{2}\right]_{2} \text { DDDEEKFLRRIGRFG }\left[(\mathrm{VPG} \mid \mathrm{G})_{2}(\mathrm{VPGKG})(\mathrm{VPG} \mid \mathrm{G})_{2}\right]_{2}\right]_{3}$} & Mineralization & 5 & $1: 3$ \\
\hline HAP-RGDS & 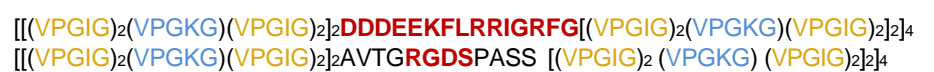 & $\begin{array}{l}\text { Mineralization and } \\
\text { cell adhesion }\end{array}$ & 5 & $1: 3$ \\
\hline
\end{tabular}



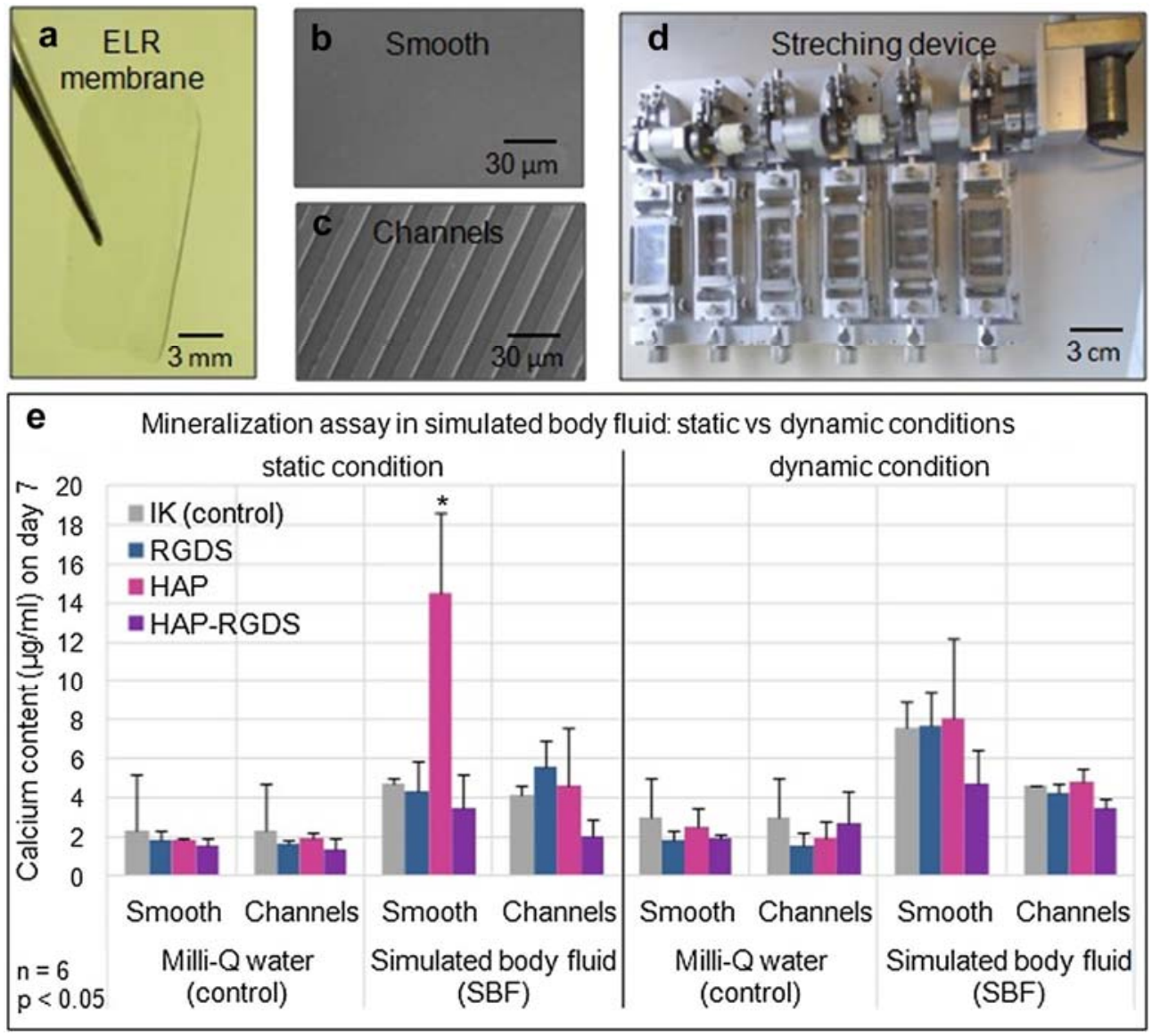

Fig. 1. (a) ELR membranes fabricated by drop-casting approach (c) exhibiting or (b) not channel topographies were tested in dynamic condition in a (d) custom-made stretching device used to apply uniform, cyclic, and uniaxial strain of 8\%. (e) Smooth HAP membranes incubated in simulated body fluid (SBF) exhibited the highest amount of deposited calcium compared to all other membranes in either static or dynamic conditions.

pulsed Bismuth liquid metal ion source $\left(\mathrm{Bi}^{3 \mathrm{p}}\right)$, at energy of $25 \mathrm{keV}$. The gun was operated with a $20 \mathrm{~ns}$ pulse width, $0.3 \mathrm{pA}$ pulsed ion current for a dosage lower than $5 \times 10^{11}$ ions $/ \mathrm{cm}^{2}$ (below the threshold level of $1 \times 10^{13}$ ions $/ \mathrm{cm}^{2}$ generally accepted for static SIMS conditions). Charge neutralization was achieved with a low energy $(20 \mathrm{eV})$ electron flood gun. The secondary ions were detected with a reflector timeof-flight analyzer, a multichannel plate (MCPs), and a time-to-digital converter (TDC). Measurements were performed with a typical acquisition time of $20 \mathrm{~s}$ at a TDC time resolution of $200 \mathrm{ps}$ and $128 \times 128$ pixel image size. Secondary ion spectra were acquired from areas of $400 \times 400 \mathrm{~mm}^{2}$ within the sample's surface. Mass spectral acquisition and image analysis were performed within the IonSpec software (version 4.1, ION-TOF, Germany) and Ion image software (version 3.1, ION-TOF, Germany), respectively. Each spectrum was normalized to the total intensity [31].

Membranes analyzed by SEM-EDS were frozen at $-80{ }^{\circ} \mathrm{C}$ overnight after washing twice with milli- $\mathrm{Q}$ water. After $24 \mathrm{~h}$ membranes were freeze-dried, and finally they were coated with a $10 \mathrm{~nm}$ layer of goldeplatinum. The mineral morphology and chemical composition were imaged and analyzed by SEM (JSM$7100 \mathrm{~F}$, JEOL, Germany) fitted with an energy dispersive X-ray microanalyzer (Oxford Inca 350 , Oxford instruments, UK) at $20 \mathrm{kV}$.

\subsubsection{Membrane Young's modulus}

The stiffness of ELR membranes was measured by the Young's modulus. A tensile test was performed under wet conditions in mili-Q water at $37^{\circ} \mathrm{C}$. ELR Membranes were fabricated in rectangular shape $\left(8 \times 15 \mathrm{~mm}^{2}\right)$. The thickness of the membranes was measured with a micrometer having a precision of $0.01 \mathrm{~mm}$. Mechanical tests were performed with a BOSE ${ }^{\circledR}$ ElectroForce ${ }^{\circledR}$ BioDynamicTM bioreactor (BOSE, Friedrichsdorf, Germany) with an orthopedic chamber, equipped with a $22 \mathrm{~N}$ loadcell at a speed of $1 \mathrm{~mm} / \mathrm{min}$, and forces were recorded using Win Test ${ }^{\circledR}$ software (BOSE, Friedrichsdorf, Germany). The Young's modulus was calculated as the slope of the straight line portion of the stressestrain curve. Membranes incubated in miliQwater were used as control.

2.6. In vitro cell differentiation

Rat MSCs obtained following a procedure described previously [38], were cultured on membranes made of IK and HAP ELR molecules comprising or not channels topographies. Cells were incubated overnight prior the application of the mechanical stimulation (Section 2.4) for 2 days. Membranes were sterilized for 20 min under ultraviolet (UV) inside the cell culture hood, and then immobilized over the silicone dishes of the stretching device. The silicone dishes were prepared by combining Elastosil component A with Elastosil component B (Elastosil RT 601; Wacker-Chemie, Germany) at a 10:1 ratio, respectively. The elastomer was mixed, poured into the dish mould and, allowed to sit at room temperature overnight. The silicone dishes were then removed, cleaned with a $1 \%$ Liqui-nox liquid detergent (Alconox Inc., White Plains, NY) in milli-Q water solution, rinsed in milli-Q water, air-dried, and autoclaved at $121^{\circ} \mathrm{C}$ for $15 \mathrm{~min}$.

Cells were cultured in osteogenic differentiation medium (OMp) in tissue culture plastic ( $\mathrm{TCPp})$ as positive control, or without dexamethasone (OM-) on ELR membranes and tissue culture plastic (TCP-) as negative control. As an additional control, ELR membranes were cultured in OM- in static conditions. OMp was prepared with $15 \mathrm{ml}$ a-Eagle minimum essential medium (aMEM, Gibco-Invitrogen, Scotland) supplemented with $10 \%$ fetal bovine serum (FBS, SigmaeAldrich, Taufkirchen, Germany), $50 \mathrm{~mm}$ ascorbic acid (Sigma, St. Louis, MO), $50 \mathrm{mg} / \mathrm{ml}$ gentamycin (Sigma, St. Louis, MO), 10 mm sodium-b-glycerophosphate (Sigma, St. Louis, MO), and $10^{-8} \mathrm{M}$ dexamethasone (Sigma, St. Louis, MO). Rat MSCs of passage 3 e6 were diluted in serum-free DMEM and seeded at $10,500 \mathrm{cells} / \mathrm{cm}^{2}$ onto the substrates. After $2 \mathrm{~h}$ of culture, all the medium was replaced with DMEM containing $10 \% \mathrm{FBS}$ for subsequent culture. Each sample was assayed in triplicate and the experiment was repeated twice.

\subsubsection{Osteoblastic differentiation and mineralization assays}

In order to analysis in vitro cell differentiation DNA content, alkaline phosphatase (ALK) activity and calcium content assays were performed on day 5 and 10 . The culture media was removed and cells were rinsed in phosphate-buffered saline (PBS). Then, $500 \mathrm{ml}$ of filter milli-Q water was added to each sample. The cell suspension was transferred to an eppendorf, sonicated for $20 \mathrm{~min}$, and the supernatant was collected and frozen at $-20^{\circ} \mathrm{C}$ overnight. The cell suspension was thawed and frozen two times more. This cell suspension was used in the following assays.

$100 \mathrm{ml}$ of the cell suspension was used to analyze the DNA concentration using the picogreen assay (Molecular Probes, The Netherlands) according to the 
manufacturer's instructions. The DNA content $(\mathrm{mg} / \mathrm{ml})$ of the samples was read in a microplate reader (BioRad 450, Bio-Rad Laboratories, Hercules, CA) at $480 \mathrm{~nm}$ and determined from a DNA standard curve. Second, in order to analyze ALK activity $100 \mathrm{ml}$ of the cell suspension was added to $100 \mathrm{ml}$ of working reagent consisting of 0.5 M 2S-amino-2methyl-l-propenyl (Sigma, St. Louis, MO), $5 \mathrm{~mm}$ p-nitro-phenol phosphate (Sigma, St. Louis, MO), and 5 mm magnesium chloride (1:1:1) (Sigma, St Louis, MO). The reaction was stopped using $100 \mathrm{ml}$ of sodium hydroxide (Sigma, St. Louis, MO), and the final absorbance was read at $405 \mathrm{~nm}$ using a microplate reade (BioRad 450, Bio-Rad Laboratories, Hercules, CA). A standard curve was generated by making serial dilutions of 4-nitrophenol, and sample measurements were extrapolated from known concentration values of the curve. ALK activity (nmol) was normalized to DNA concentration $(\mathrm{mg} / \mathrm{ml})$. Finally, information about the mineral ized matrix formation on the ELR membranes was obtained by the calcium conten assay using $20 \mathrm{ml}$ of the cell suspension. The calcium content $(\mathrm{mg} / \mathrm{ml})$ was deter mined using the protocol described in Section 2.5.1, and normalized to DNA concentration $(\mathrm{mg} / \mathrm{ml})$.

\subsubsection{Scanning electron microscopy characterization}

Cells cultured on ELR membranes were rinsed twice with filtered PBS, fixed with $2 \%$ glutaraldehyde (Sigma, St. Louis, MO) in cacodylate (Sigma, St. Louis, MO) buffer for $5 \mathrm{~min}$, and rinsed twice with $0.1 \mathrm{M}$ sodium cacodylate buffer for $5 \mathrm{~min}$. Then, the samples were dehydrated in a series of $70 \%, 80 \%, 90 \%, 96 \%$, and $100 \%$ ethanol so lution. Finally, two drops of tetramethylsilane (Sigma, St. Louis, MO) were added to each sample followed by air-dried for $5 \mathrm{e} 10 \mathrm{~min}$. The samples were coated with a $10 \mathrm{~nm}$ layer of gold.

\subsection{In vivo characterization}

The bioactivity of Smooth HAP ELR membrane scaffolds was tested in a $5 \mathrm{~mm}$ critical-size rat calvarial defect model [32]. The rats were treated with HAP mem branes to evaluate the effect of the bioactive sequence, IK membranes to evaluate the effect of non-bioactive sequence and without treated to evaluate the healing in the empty defect. Membranes were sterilized in UV for 20 min and hydrated in physiological saline, previously to fill the calvarial defect.

A total of 21 male SpragueeDawley rats (12-week-old, 425e475 g) were obtained from Janvier Lab Animal (France) (7 rats per group). The animals were housed singly and received food and water ad libitum. All animals' research protocols were approved by the Ethical Committee of Animal Experimentation (CEEA) of the University of Barcelona (Spain) and the Ministry of Agriculture, Livestock, Fisheries, Food, and the Environment of the Government Institution of Catalonia (Spain).

\subsubsection{Surgical procedure}

The surgery was performed in the Laboratory Animal Applied Research Platform at Parc Cientific Barcelona (PCB, Spain). The animals were anaesthetized using isoflurane (SigmaeAldrich, Spain) inhalation anesthesia (5\% induction; $3 \%$ mainte nance). All animals prior to the surgical intervention received an intraperitonea injection of buprenorphine $(0.05 \mathrm{mg} / \mathrm{kg})$ for postoperative analgesia, and its administration was repeated $12 \mathrm{e} 16 \mathrm{~h}$ after the surgery. The animals were positioned onto a heating pad $\left(37^{\circ} \mathrm{C}\right)$ during all the surgery process. The skin that covered the skull was shaved and disinfected with iodopovidone solution. A midline incision of $2 \mathrm{~cm}$ was performed with a surgical blade. The skin was then reflected bilaterally to expose the calvarial bone surface. A $5 \mathrm{~mm}$-diameter trephine bur (Fine Science tools, Heidelberg, Germany) mounted on a dental hand piece (Foredom, Bethel, New York, USA) was used to drill a round, segmental defect in the right parietal bone. During the drilling, the area was continuously irrigated with sterile saline solution, and subsequently the calvarial disk was carefully removed and extracted using tweezers. The defect was rinsed with physiological saline and the hydrated ELR membrane
$(7.5 \times 7.5 \mathrm{~mm})$ was implanted covering the defect. The extreme of the membrane

was fixed to the bone applying a drop of cyanoacrylate .The skin was closed over in layers with 4.0 Nylon sutures and was applied iodopovidone solution to prevent infections. Animals were monitored daily until euthanasia for any complications or abnormal behavior and were sacrificed 36 days after surgery by carbon dioxide inhalation.

\subsubsection{High-resolution micro-computed tomography analysis}

Immediately after rats were sacrificed, a high-resolution micro-computed tomography (microCT) analysis was performed using the Skyscan 1172 computed microtomographic system (Kontich, Belgium). The parameters of the scan were voltage source $81 \mathrm{kV}$, current source $124 \mathrm{~mA}$, image pixel size $9 \mathrm{~mm}$, an aluminum filter of $0.5 \mathrm{~mm}$, a tomographic rotation of $180^{\circ}$ and a sample rotation step of $0.8^{\circ}$. The reconstruction was carried out with NRecon (v1.6.2.0) software using a specific post-alignment per each sample and applying a medium intensity ring artefact correction. Microtomographic 3D analysis was analyzed with CTAn (v.1.10.1.3) software, using a global threshold of 50e255. A cylindrical volume of interest (VOI) was used to quantify the bone volume and bone mineral density corresponding with the size of the defect.
2.7.3. Histological analysis

The calvarias were extracted from the skull and fixed in $4 \%$ paraformaldehyde (Panreac, Spain) at $\mathrm{pH} 1 / 47.2$ for 2 days followed by bone decalcification with Sur gipath Decalcifier II (Leica biosystems, Spain) for $4 \mathrm{~h}$. Next, the calvarias were embedding in paraffin and $3 \mathrm{~mm}$ sections were made using a microtome. The his tological sections were stained with H\&E and Trichrome Goldner and observed with a microscope Zeiss AxioScope A (Carl Zeiss, Madrid, Spain) incorporating a Zeiss AxioCam MRc5 camera (Carl Zeiss, Madrid, Spain) for qualitative and semiquantitative evaluation. A score system was assigned ( 0 , absent; 1 , scarcely present 2 , slightly present 3 , present; 4 , intensively present) to the new ossified tissue (os teoblasts embedded in osteoid matrix) observed on the edge and in the middle of the defect allowing a numerical comparison.

\subsection{Statistics}

Data were reported as mean \pm standard error of the mean. Statistical analysis was performed by one-way analysis of variance using ANOVA and student $t$-test. The non-parametric KrusskalleWallis test was carried out to compare the histological semiquantitative results among groups for the in vivo experiment. Finally, $p$ values $<0.05$ were considered significant.

\section{Results}

\subsection{ELR membrane fabrication}

Membranes were fabricated as previously reported [25,27] from either ELR materials comprising a bioactive sequence (RGDS, HAP or HAP-RGDS) or a non-bioactive ELR material (IK) used as control (Table 1). The quality and reproducibility of the membranes exhibiting topographical channels were verified by profilometry and SEM observations. These results confirmed topographical patterns with well-defined features that closely resembled those of the PDMS molds.

\subsection{In vitro membrane mineralization}

ELR membrane mineralization in vitro was assessed using a variety of techniques. First, the calcium (Ca) content assay revealed the highest amount of deposited $\mathrm{Ca}$ on Smooth HAP membranes tested in static condition $(14.49 \pm 4.19 \mathrm{mg} / \mathrm{ml})$ compared to all other membranes in either static or dynamic conditions (Fig. 1e). Second, TOF-SIMS analysis confirmed the presence of calcium phosphate (CaP) mineral on these membranes. As expected, positive TOF-SIMS peaks demonstrated the presence of $\mathrm{CH}_{4} \mathrm{~N}^{\mathrm{p}}$ (blue) on all ELR membranes, which corresponds to the common amino acid sequence of all ELRs tested. However, only HAP membranes exhibited peaks for $\mathrm{Ca}^{2 \mathrm{p}}$ (green) and $\mathrm{CaOH}^{\mathrm{p}}$ (red) (Fig. 2ced), which corroborate the results of the Ca content assay. Similarly, negative TOF-SIMS peaks also revealed signals for $\mathrm{CN}^{-}$and $\mathrm{CNO}^{-}$ on all ELR membranes, which again correspond to the common amino acid sequence of all tested ELRs, while signals for $\mathrm{PQ}^{-}$and $\mathrm{PO}_{3}^{-}$were only observed on Smooth HAP membranes (Fig. 2eef). The presence of $\mathrm{CaP}$ was also confirmed by chemical images that indicated strong $\mathrm{PO}_{2}^{-}$and $\mathrm{PO}_{3}^{-}$signals only on Smooth HAP membranes (Fig. 2geh). Furthermore, the total ion image revealed a homogenous distribution of the deposition of mineral within the ELR membrane surface. Finally, SEM examination (Fig. 2aeb) and EDS analysis (Fig. 2iej) confirmed the presence of mineral on Smooth HAP membranes with a $\mathrm{Ca} / \mathrm{P}$ ratio of 1.78 .

\subsection{Young's modulus}

Tensile tests were conducted to investigate the effect of mineralization on the mechanical properties of the different membranes. Smooth HAP membranes were incubated in SBF for 7 days in static condition displayed the highest Young's modulus (E) $(2081 \pm 315 \mathrm{kPa})$ compared to all other tested membranes (Fig. $2 \mathrm{k})$, which exhibited moduli ranging between $362 \pm 74$ and $600 \pm 249 \mathrm{kPa}$ (Supplementary data Table 1). 

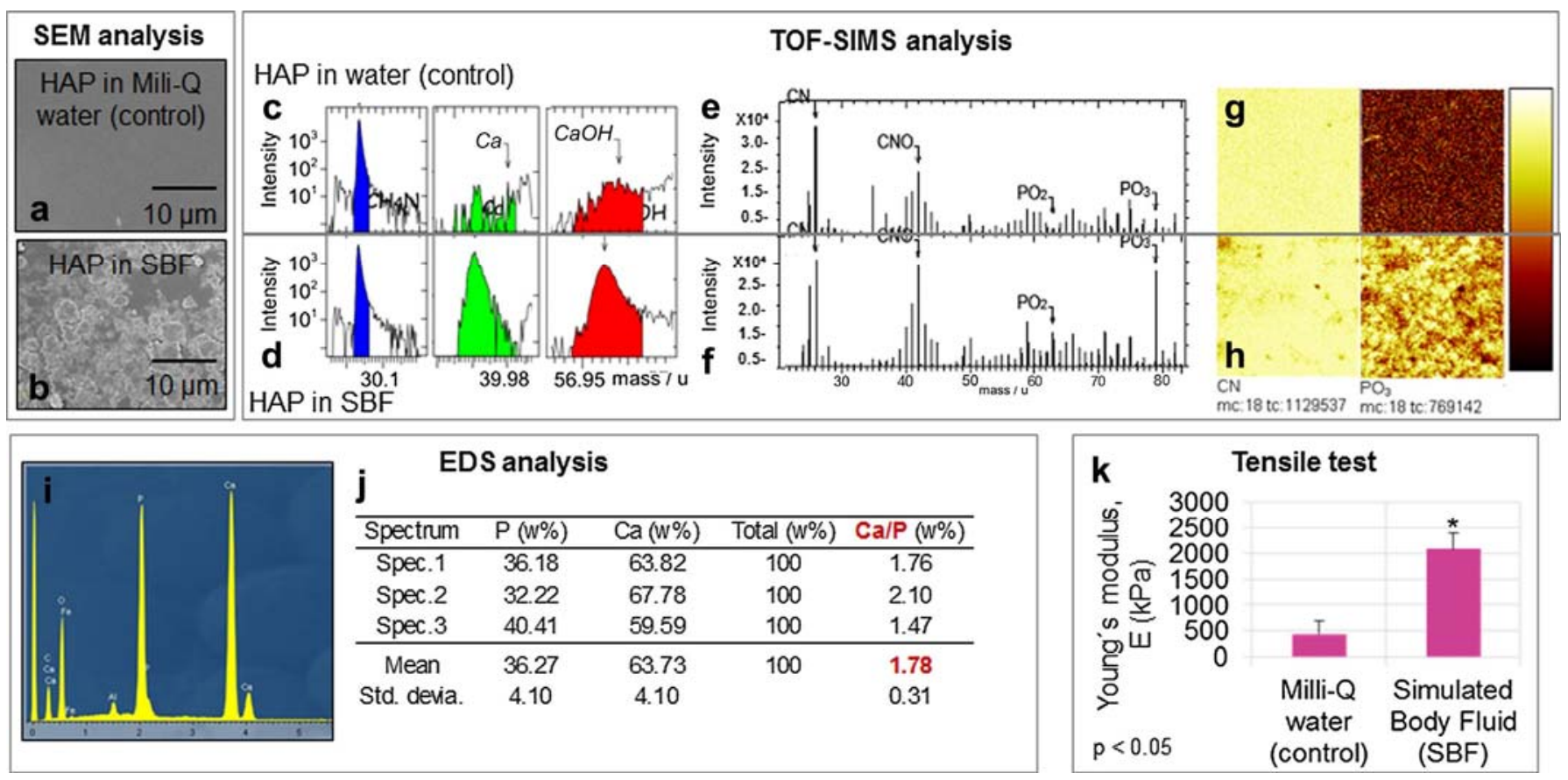

Fig. 2. Presence of mineral was observed by SEM examination on (b) Smooth HAP membrane incubated in SBF in static conditions compared with (a) the control in mili-Q water. (c) TOF-SIMS revealed in both cases peaks of $\mathrm{CH}_{4} \mathrm{~N}^{\mathrm{p}}$ and $\mathrm{CN}^{-}$corresponding with amino acid from ELR molecules. While peaks of $\mathrm{Ca}^{2 \mathrm{p}}$ (green), $\mathrm{CaOH}^{\mathrm{p}}$ (red), $\mathrm{PO}_{2}^{-}$and $\mathrm{PO}_{3}^{-}$correspond with the mineral observed on Smooth HAP membranes incubated in SBF compared with the control in mili-Q water. (d) EDS analysis confirmed that the mineral exhibited on Smooth HAP membranes displayed a $\mathrm{Ca} / \mathrm{P}$ proportion of 1.78. (e) Additionally, the highest value of Young modulus was observed on these membranes incubated in SBF. (For interpretation of the references to color in this figure legend, the reader is referred to the web version of this article.)
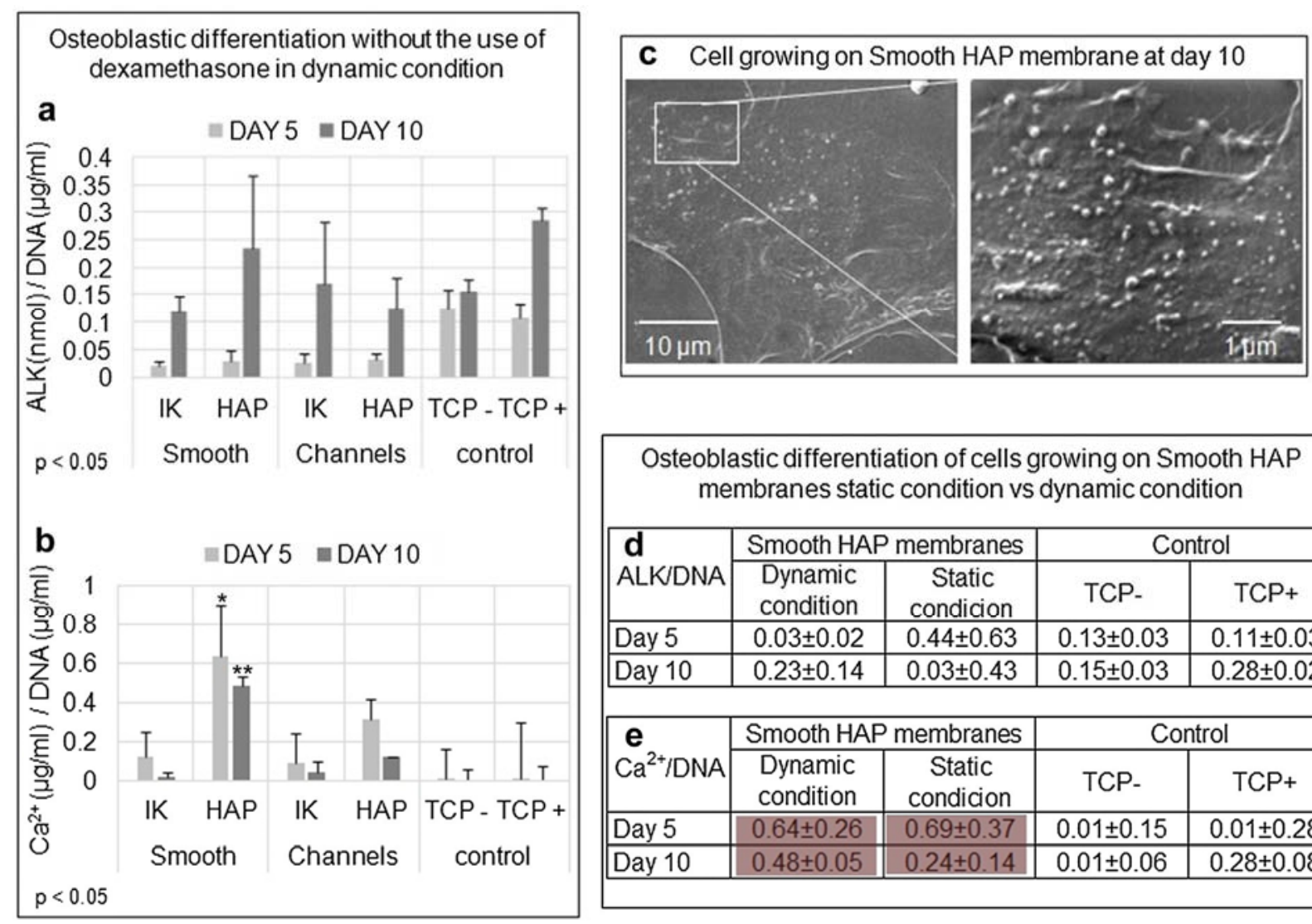

\begin{tabular}{|c|c|c|c|c|}
\hline \multicolumn{5}{|c|}{$\begin{array}{l}\text { Osteoblastic differentiation of cells growing on Smooth HAP } \\
\text { membranes static condition vs dynamic condition }\end{array}$} \\
\hline \multirow{2}{*}{$\begin{array}{l}\mathbf{d} \\
\text { ALKJDNA }\end{array}$} & \multicolumn{2}{|c|}{ Smooth HAP membranes } & \multicolumn{2}{|c|}{ Control } \\
\hline & $\begin{array}{l}\text { Dynamic } \\
\text { condition }\end{array}$ & $\begin{array}{c}\text { Static } \\
\text { condicion }\end{array}$ & TCP- & $\mathrm{TCP}+$ \\
\hline Day 5 & $0.03 \pm 0.02$ & $0.44 \pm 0.63$ & $0.13 \pm 0.03$ & $0.11 \pm 0.03$ \\
\hline Day 10 & $0.23 \pm 0.14$ & $0.03 \pm 0.43$ & $0.15 \pm 0.03$ & $0.28 \pm 0.02$ \\
\hline \multirow{2}{*}{$\begin{array}{l}\mathbf{e} \\
\mathrm{Ca}^{2+} / \mathrm{DNA}\end{array}$} & \multicolumn{2}{|c|}{ Smooth HAP membranes } & \multicolumn{2}{|c|}{ Control } \\
\hline & $\begin{array}{l}\text { Dynamic } \\
\text { condition }\end{array}$ & $\begin{array}{c}\text { Static } \\
\text { condicion }\end{array}$ & TCP- & $\mathrm{TCP}+$ \\
\hline Day 5 & $0.64 \pm 0.26$ & $0.69 \pm 0.37$ & $0.01 \pm 0.15$ & $0.01 \pm 0.28$ \\
\hline Day 10 & $0.48 \pm 0.05$ & $0.24 \pm 0.14$ & $0.01 \pm 0.06$ & $0.28 \pm 0.08$ \\
\hline
\end{tabular}

Fig. 3. (a) The highest ALK/DNA expression and (b) Ca deposition was observed on rMSCs growing on Smooth HAP membranes in non-osteogenic differentiation medium, compared to cells on any other membrane or control substrate growing in osteogenic differentiation medium. (c) SEM observations confirmed the presence of this mineral on cells cultured on Smooth HAP membranes on day 10. ALK activity (d) and Ca deposition (e) between cell growth on membranes tested in static or dynamic culture conditions were not significantly different. 


\subsection{In vitro cell differentiation}

Given the observed mineralizing nature of the Smooth HAP membranes, osteoblastic differentiation of rMSCs was investigated in both static and dynamic conditions by measuring ALK and $\mathrm{Ca}$ deposition. On day 5 and 10, the highest ALK/DNA expression was observed on cells growing on Smooth HAP membranes in nonosteogenic differentiation medium $(0.23 \pm 0.13)$, which was statistically similar to that expressed by cells growing on tissue culture plastic in osteogenic differentiation medium (TCPp) $(0.28 \pm 0.02)$ (Fig. 3a). Furthermore, cells on these membranes growing in nonosteogenic differentiation medium also exhibited the highest $\mathrm{Ca}$ deposition on both day 5 and 10 compared to cells on any other membrane or control substrate growing in osteogenic differentiation medium (Fig. 3b). SEM observations confirmed the presence of mineralized extracellular matrix formation on cells cultured on Smooth HAP membranes on day 10 (Fig. 3c). Finally, there were no significant differences in ALK activity and Ca deposition between cells growing on membranes tested in static or dynamic culture conditions (Fig. 3dee).

\subsection{In vivo characterization}

Given the observed in vitro mineralizing nature and enhancement of osteoblastic differentiation of Smooth HAP membranes, experiments were conducted to analyze their bone regeneration capacity in vivo using an orthotopic critical-size rat calvarial defect model (Fig. 4aeb).

\subsubsection{Localization of ELR membranes within the defect site}

In order to confirm that the ELR membranes were stable and positioned within the defect site, three animals were implanted with ELR membranes and sacrificed on day 7. In all three animals, the membranes were observed to be positioned within the defect site in the same location as they were placed during implantation (Fig. 4c).

\subsubsection{Quantification of bone formation by microCT}

The microCT analysis demonstrated that the animals implanted with the HAP membranes presented the highest mean volume of ossified tissue within the defect $\left(12.6 \pm 2.4 \mathrm{~mm}^{3}\right)$ (Fig. $4 \mathrm{~d}, \mathrm{f}$ ) compared to animals receiving the non-bioactive IK membranes $\left(9.2 \pm 2.6 \mathrm{~mm}^{3}\right)($ Fig. $4 \mathrm{~g})$ and those left untreated $\left(9.0 \pm 1.4 \mathrm{~mm}^{3}\right)$ (Fig. 4h). While some regeneration was observed on all tested groups along the rim area of the defect, only animals implanted with the HAP membrane exhibited ossified tissue towards the center of the defect tending to breach the critical-size gap. These results were confirmed by histological analysis (Section 3.4.3). MicroCT data was also used to quantify bone mineral density within the defect. In this case, there was greater variation between
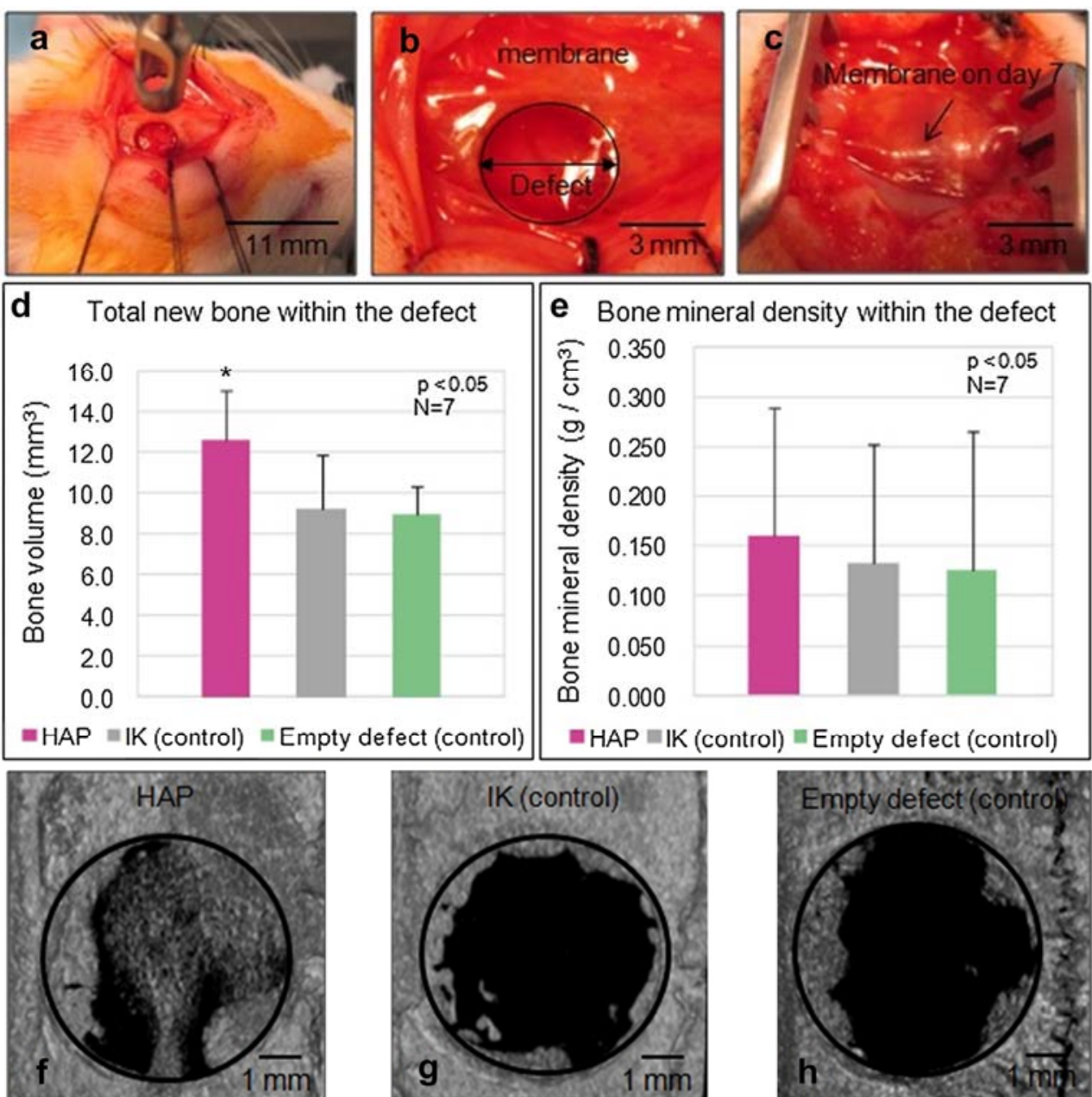

Fig. 4. (a) An orthotopic critical-size rat calvarial defect model was used to analyze the bone regeneration capacity of (b) Smooth HAP membranes. (c) Membranes were observed to be positioned within the defect site in the same location as they were placed during implantation on day 7 . The microCT analysis demonstrated that animals implanted with the (d) HAP membranes presented the highest mean volume of ossified tissue (f) within the defect compared to animals receiving the (g) non-bioactive IK membranes and (h) those left untreated. (e) MicroCT analysis of bone mineral density within the defect revealed no significant differences between the tested groups. 
samples and no significant differences were observed between the three tested groups (Fig. 4e).

\subsubsection{Quantification of bone formation by histological analysis}

Histological sections stained with H\&E indicated greater ossification on animals treated with the HAP membranes both around the rim and in the middle of the defect (Fig. 5a). Within these regions, Trichrome Goldner staining (Fig. 5b) demonstrated the presence of both immature (osteoid) and mature bone as well as a layer of active osteoblasts (Fig. 5c) embedded within an osteoid matrix located on the growth front along the developing bone around the rim of the defect (Fig. 5d). In contrast, animals that were implanted with the IK membranes and animals that were not treated with any membrane only exhibited bone formation around the edge of the defect. In addition, in accordance with the MicroCT results, semi-quantitative analysis of histological sections also verified the presence of more regeneration in animals implanted with the HAP membranes compared to the other groups (Fig. 5e).

\section{Discussion}

Many proteins found in bone have the capacity to modulate or inhibit mineralization in vivo [32]. The possibility to fabricate materials using proteins as both structural and functional buildingblocks may provide an effective tool to improve bone regeneration with high efficiency and control. In previous work, we developed a fabrication process to reproducibly create robust and tunable ELR membranes that exhibit specific physical and biomolecular signals [27] and the capacity to promote osteoblastic differentiation in vitro [25]. The main objective of the present study was to determine the in vitro mineralization and in vivo bone regeneration potential of bioactive ELR membranes in an animal model. In particular, the focus was to assess the bioactivity of ELR membranes containing the amino acid sequence DDDEEKFLRRIGRFG (HAP). This segment corresponds to $\mathrm{SN}_{\mathrm{A}} 15$, an analog of the SN15 fragment of statherin in which aspartate (D) residues substitute the original phosphoserines and whose bioactivity is equivalent to the SN15 fragment of statherin [33]. Statherin is a protein found in saliva, a supersaturated metastable solution compound of ions, proteins, and water, which plays a role in the nucleation and growth of hydroxyapatite in the oral environment [34]. The N-terminal of the SN15 segment of statherin is formed by a sequence of negatively charged residues such as aspartic acid (D), glutamic acid (E) and phosphorylated-serine (S(P)) [33]. This region is responsible for binding calcium ions present in the oral environment and stabilizing the growth and critical size of the $\mathrm{CaP}$ cluster required for precipitation and transformation into a crystalline phase of hydroxyapatite [35]. Upon a pH decrease next to the enamel due to the presence of metabolic products secreted by bacteria, statherin releases the $\mathrm{CaP}$ ions on the surface of the tooth and enables their use to promote remineralization [36]. Our hypothesis was that the use of ELRs exhibiting this $\mathrm{SN}_{\mathrm{A}} 15$ peptide sequence, which is known to modulate mineralization in enamel as described above, may be used to enhance mineralization in bone. In order to further enhance membrane bioactivity, we also designed
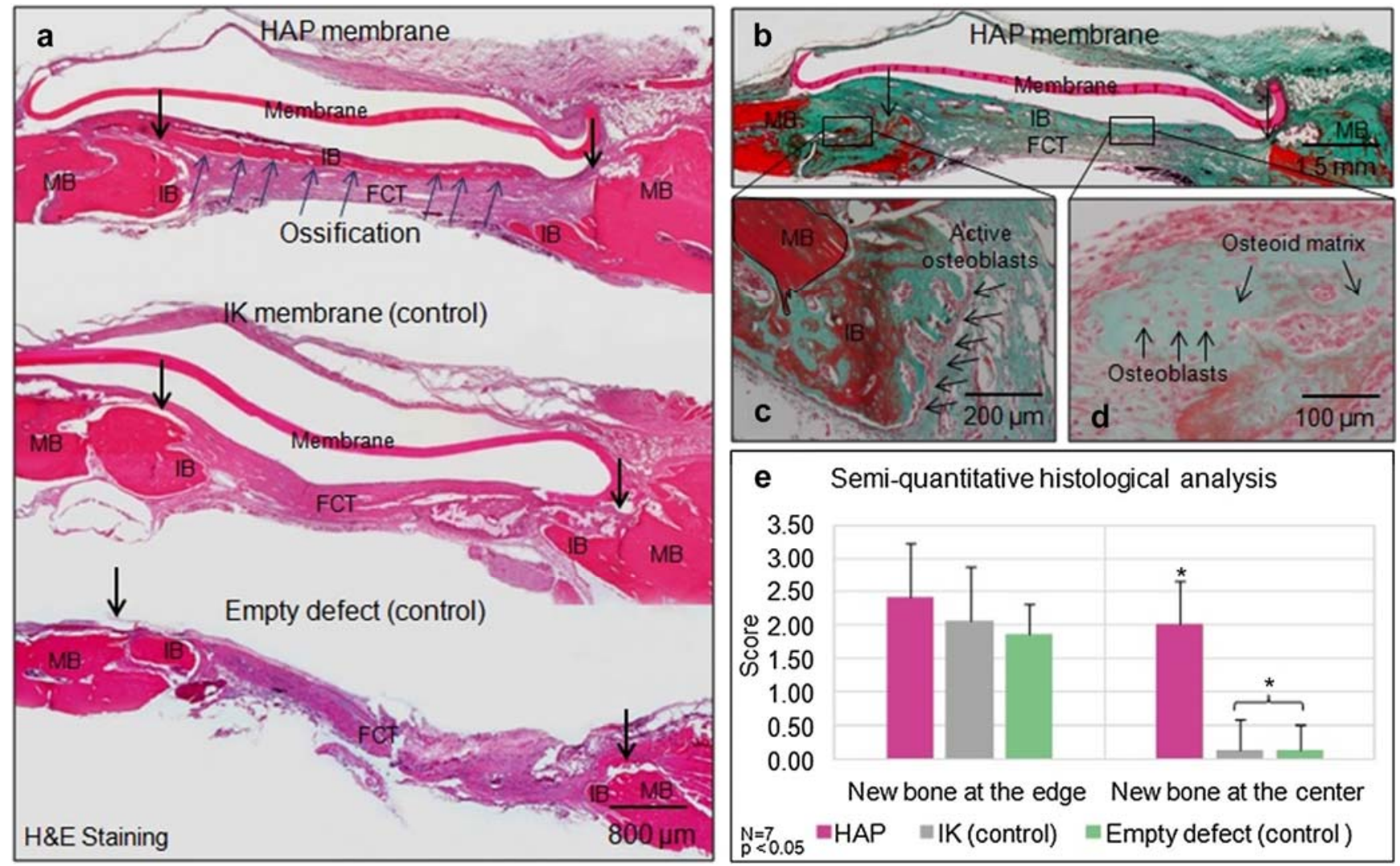

Fig. 5. (a) Histological sections stained with H\&E indicated greater ossification on animals treated with the Smooth HAP membranes (blue arrows) both around the rim and in the middle of the defect compared to animals receiving the non-bioactive IK membranes and those left untreated. (b) Histological sections stained with Trichrome Goldner of rats treated with Smooth HAP membranes demonstrated (c) the presence of immature (osteoid) and mature bone as well as (d) a layer of active osteoblasts embedded within an osteoid matrix located on the growth front along the developing bone around the rim of the defect. (e) Semi-quantitative analysis of histological sections verified the presence of more regeneration in animals implanted with the HAP membranes compared to the other groups ( $P$ 1/4 0.03. (FCT: fibrous connective tissue, MB: mature bone, IB: immature bone). (For interpretation of the references to color in this figure legend, the reader is referred to the web version of this article 

and used ELRs with an additional RGDS sequence (HAP-RGDS) in order to promote integrin-mediated cell adhesion.

Mineralization experiments, with and without cells, were con- ducted in simulated body fluid (SBF) [29] in both static and dynamic experimental conditions since some studies have reported an increase of cell-mediated mineralization under applied strain [30]. In this work, Smooth HAP membranes tested under static conditions reproducibly and significantly increased their mineral- ization compared to all other tested membranes as revealed by the calcium content assay (Fig. 1e), SEM observations (Fig. 2a, b), TOF- SIMS analysis (Fig. 2c, d), and EDS measurements (Fig. 2i, j). These HAP membranes also exhibited a significant increase in Young's modulus compared to all other tested membranes (Fig. 2k and Supplementary data Table S1), an expected result given the pres- ence of the observed mineral layer. These results are consistent with studies demonstrating the affinity of the $\mathrm{SN}_{\mathrm{A}} 15$ fragment to adsorb to hydroxyapatite surfaces by chelation with surface cal- cium ions in vitro [37]. This affinity depends on the number and close vicinity of the negatively charged residues in the $\mathrm{N}$-terminal domain, as demonstrated by studies using the individual $\mathrm{SN}_{\mathrm{A}} 15$ sequence [33] and within our HAP ELR molecule on titanium sur- faces [38]. Therefore, it is possible that the mineralization observed on our HAP membranes was produced by the high density of negative charges present on the N-terminal segment of the $\mathrm{SN}_{\mathrm{A}} 15$ fragment of our HAPcontaining ELRs. This hypothesis would explain the lower mineralization observed on HAP-RGDS mem- branes, since these membranes contain a lower density of the $\mathrm{SN}_{\mathrm{A}} 15$ sequence compared to HAP membranes [25].

Despite the strong mineralization on Smooth HAP membranes

in static conditions, HAP membranes with Channel topographies and Smooth HAP membranes in dynamic conditions exhibited lower $\mathrm{CaP}$ deposition. While the reason for this decrease is not yet clear, it is possible that the $8 \%$ uniaxial strain used in this study may have assisted the release of the mineral from the membranes due to mechanical deformation. An alternative explanation might be related with the decrease of mineralization as a result of a decrease in wettability [39] due to the presence of the channel micro- topographies. Further studies will have to be performed in order to confirm this hypothesis.

Remarkably, in both static and dynamic conditions, rMSCs growing on HAP membranes in non-osteogenic differentiation medium exhibited similar levels of ALK/DNA expression and higher Ca deposition compared to cells growing in osteogenic differenti- ation medium on tissue culture plastic (TCPp). These results are in accordance with our previous finding that the amino acid sequence of the $\mathrm{SN}_{\mathrm{A}} 15$ fragment significantly upregulates the early osteo- blastic marker osterix in vitro even in the absence of osteogenic differentiation medium [25]. Previous studies have reported that the application of cyclic uniaxial strain on MSCs increased the production of matrix mineralization [40]. However, in our study mineralization of the membrane in the presence of cells was similar for both static and dynamic culture conditions. It is possible that the mineralizing effect expected to arise from the applied strain was masked by the strong mineralizing effect of the $\mathrm{SN}_{\mathrm{A}} 15$ frag- ment present in HAP membranes.

Due to the strong osteoblastic differentiation and mineralization observed in the Smooth HAP membranes in vitro, they were subsequently implanted and assessed in a critical-size rat calvarial model. At day 7, membrane localization were assessed and confirmed to be within the defect in the same place as they were positioned at the time of implantation. This stability results from the distinctive strength and ease of manipulation exhibited by our ELR membranes, which contrast the inherent weakness of other peptide and protein- based scaffolds. With respect to membrane bioactivity, animals implanted with HAP membranes had the highest mean volume of 
ossified tissue exhibiting an osteoid matrix with active osteoblasts within the defect and in cases even bridging across the critical-size defect (Figs. 4, 5c and 5d). Bone mineral density was also measured by microCT although the results exhibited a high variability and did not demonstrate significant differences. This high variability may have resulted because the values of bone mineral density obtained for all samples were lower than the selected range of the accuracy of the equipment. Nonetheless, altogether the results are consistent with events normally observed in natural intramembranous ossifi- cation [41] and in accordance with studies that have demonstrated bone regeneration using other peptide-based materials with mineralization-promoting signals [6]. Both our in vitro data and in vivo results suggest that the presence of the $\mathrm{SN}_{\mathrm{A}} 15$ sequence of HAP ELRs may promote early biomineralization and potentially lead to cellular signaling that stimulates progenitor cells and enhances the growth of osteoblasts in vivo.

The main design feature of the present study was the generation of ELR building-blocks that offer both mechanical stability through the elastin-like sequences and molecular signaling leading to min- eral formation and osteogenesis through the $\mathrm{SN}_{\mathrm{A}} 15$ segment. ELR- based materials have been found non-cytotoxic and bioactive [42] when used in ostechondral [43] and vascular [44] defects. To our knowledge, this is the first study that demonstrates enhanced bone regeneration using ELRs and the use of a statherin analog known to modulate mineralization in enamel for bone regeneration. A po- tential explanation for this finding may lie in the fact that statherin found in saliva and proteins found in bone, all of which regulate the growth of CaP, descend from a set of genes that have a common ancestor [45]. This relation may help explain why the $\mathrm{SN}_{\mathrm{A}} 15$ frag- ment found to control enamel mineralization [36], is also efficiently promoting bone formation as observed in this study. The use of non- collagenous proteins known to regulate the remarkable mineral formation found in enamel in order to design materials to improve mineralization and bone regeneration is an exciting possibility.

\section{Conclusions}

We report on the enhanced mineralization, osteogenesis and in vivo bone regeneration properties of molecularly designed ELR membranes. Smooth membranes, containing an analog of the SN15 fragments of statherin (DDEEKFLRRIGRFG), exhibited the highest quantity of calcium phosphate $(\mathrm{Ca} / \mathrm{P}$ in 1.78) deposition with and without cells compared to all other tested membranes. Furthermore, these membranes displayed the highest production of alka- line phosphatase (ALK) on day 10 even in the presence of nonosteogenic media. This strong bioactivity was further demon- strated in vivo as animals implanted with theses membranes exhibited the highest bone volume within the defect. This study validates the ability of generating ELR molecules that can serve as structural and functional building blocks to create robust bio- materials capable of orchestrating biological responses. Thin robust membranes made completely from molecularly designed ELRs that are capable to promote osteogenesis and enhance mineralization could serve as effective periosteal grafts capable of enhancing bone regeneration.

\section{References}

[1] Lienemann P, Lutolf M, Ehrbar M. Biomimetic hydrogels for controlled biomolecule delivery to augment bone regeneration. Adv Drug Deliv Rev 2012;64:1078e89.

[2] Brown R. Direct collagen-material engineering for tissue fabrication. Tissue Eng Part A 2013;19:1495e8.

[3] Collins M, Birkinshaw C. Hyaluronic acid based scaffolds for tissue engineeringea review. Carbohydr Polym 2013;92:1262e79.

[4] Ana MM, Catarina MA, Kasper FK, Antonios GM, Rui LR. Responsive and in situ-forming chitosan scaffolds for bone tissue engineering applications: an overview of the last decade. J Mater Chem 2010;20:1638e45.

[5] Mobini S, Hoyer B, Solati-Hashjin M, Lode A, Nosoudi N, Samadikuchaksaraei A, et al. Fabrication and characterization of regenerated silk scaffolds reinforced with natural silk fibers for bone tissue engineering. J Biomed Mater Res A 2013;101:2392e404.

[6] Mata A, Geng Y, Henrikson K, Aparicio C, Stock S, Satcher R, et al. Bone regeneration mediated by biomimetic mineralization of a nanofiber matrix. Biomaterials 2010;31:6004e12.

[7] Zhang X, Awad H, O'Keefe R, Guldberg R, Schwarz E. A perspective: engineering periosteum for structural bone graft healing. Clin Orthop Relat Res 2008;466:1777e 87.

[8] Gkioni K, Leeuwenburgh S, Douglas T, Mikos A, Jansen J. Mineralization of hydrogels for bone regeneration. Tissue Eng Part B Rev 2010;16:577e85.

[9] Chen Y-J, Chung M-C, Jane Yao C-C, Huang C-H, Chang H-H, Jeng J-H, et al. The effects of acellular amniotic membrane matrix on osteogenic differentiation and ERK1/2 signaling in human dental apical papilla cells. Biomaterials 2012;33:455e 63 .

[10] Lee E-J, Shin D-S, Kim H-E, Kim H-W, Koh Y-H, Jang J-H. Membrane of hybrid chitosan-silica xerogel for guided bone regeneration. Biomaterials 2009;30: $743 \mathrm{e} 50$.

[11] Kim K-H, Jeong L, Park H-N, Shin S-Y, Park W-H, Lee S-C, et al. Biological efficacy of silk fibroin nanofiber membranes for guided bone regeneration. J Biotechnol 2005;120:327e39.

[12] Mota J, Yu N, Caridade S, Luz G, Gomes M, Reis R, et al. Chitosan/bioactive glass nanoparticle composite membranes for periodontal regeneration. Acta Biomater 2012;8:4173e80.

[13] Douglas T, Gassling V, Declercq H, Purcz N, Pamula E, Haugen H, et al. Enzymatically induced mineralization of platelet-rich fibrin. J Biomed Mater Res A 2012;100:1335e 46 .

[14] Yang F, Both S, Yang X, Walboomers X, Jansen J. Development of an electrospun nano-apatite/PCL composite membrane for GTR/GBR application. Acta Biomater 2009;5:3295e304.

[15] Kharaziha M, Fathi M, Edris H. Development of novel aligned nanofibrous composite membranes for guided bone regeneration. J Mech Behav Biomed Mater 2013;24:9e20.

[16] Benedetto M, Chiara EG, Jake EB, Showan NN. Collagen gel fibrillar density

dictates the extent of mineralization in vitro. Soft Matter 2011;7:9898e907.

[17] Schneider D, Weber F, Grunder U, Andreoni C, Burkhardt R, Jung R. A randomized controlled clinical multicenter trial comparing the clinical and histological performance of a new, modified polylactide-co-glycolide acid membrane to an expanded polytetrafluorethylene membrane in guided bone regeneration procedures. Clin Oral Implants Res 2014;25(2):150e9. 
[18] Shin S-I, Herr Y, Kwon Y-H, Chung J-H. Effect of a collagen membrane com- bined with a porous titanium membrane on exophytic new bone formation in a rabbit calvarial model. J Periodontol 2013;84:110e6.

[19] Wang Y, Shi R, Gong P, Li J, Li J, Ao D, et al. Bioelectric effect of a chitosan bioelectret membrane on bone regeneration in rabbit cranial defects. J Bioact Compat Polym 2012;27(2):122e32.

[20] Gentile P, Chiono V, Tonda-Turo C, Ferreira A, Ciardelli G. Polymeric mem- branes for guided bone regeneration. Biotechnol J 2011;6:1187e97.

[21] Gungormus M, Branco M, Fong H, Schneider J, Tamerler C, Sarikaya M. Self assembled bi-functional peptide hydrogels with biomineralization-directing peptides. Biomaterials 2010;31:7266e 74

[22] Sílvia G, Isabel BL, João FM, Rui LR, David LK. Spider silk-bone sialoprotein fusion proteins for bone tissue engineering. Soft Matter 2011;7:4964e73.

[23] Joaquim SB, Artur R, Ana MT, Matilde A, Francisco JA, Jose CR-C, et

al.

Development of biomimetic chitosan-based hydrogels using an elastinlike Polymer. Adv Eng Mater 2010;12:37e44.

[24] Girotti A, Reguera J, Rodríguez-Cabello J, Arias F, Alonso M, Matestera A. Design and bioproduction of a recombinant multi(bio)functional elastinlike protein polymer containing cell adhesion sequences for tissue engineering purposes. J Mater Sci Mater Med 2004;15:479e84.

[25] Tejeda-Montes E, Smith K, Rebollo E, Gomez R, Alonso M, Rodríguez-Cabello J et al. Bioactive membranes for bone regeneration applications: effect of physical and biomolecular signals on mesenchymal stem cell behaviour. Acta Biomater 2014;10:134e41.

[26] Ana CM, Katherine HS, Esther T-M, Elisabeth E, Rui LR, Helena SA, et al. $\mathrm{Co}^{-}$Assembled and microfabricated bioactive membranes. Adv Funct Mater 2013;23:430e8.

[27] Tejeda-Montes E, Smith K, Poch M, Lopez-Bosque M, Martín L, Alonso M, et al. Engineering membrane scaffolds with both physical and biomolecular signaling. Acta Biomater 2012;8:998e1009.

[28] Prodanov L, te Riet J, Lamers E, Domanski M, Luttge R, van Loon J, et al The interaction between nanoscale surface features and mechanical loading and its effect on osteoblast-like cells behavior. Biomaterials 2010;31:7758e65.

[29] Kokubo T, Takadama H. How useful is SBF in predicting in vivo bone bioac- tivity? Biomaterials 2006;27:2907e15.

[30] Winter L, Walboomers X, Bumgardner J, Jansen J. Intermittent versus continuous stretching effects on osteoblast-like cells in vitro. J Biomed Mater Res A 2003;67:1269e75.

[31] Sebastian M, Perez R, Egido J. Use of TOF-SIMS in vascular biology. In Vivanco F, editor. Vascular proteomics: methods and protocols. Methods in molecular biology, New York, vol. 1000; 2013. pp. 33e43. chapter 3.

[32] Benesch J, Mano J, Reis R. Proteins and their peptide motifs in acellular apatite mineralization of scaffolds for tissue engineering. Tissue Eng Part B Rev 2008;14:433e45.

[33] Raj P, Johnsson M, Levine M, Nancollas G. Salivary statherin. Dependence on sequence, charge, hydrogen bonding potency, and helical conformation for adsorption to hydroxyapatite and inhibition of mineralization. J Biol Chem 1992;267:5968e76.

[34] Stayton P, Drobny G, Shaw W, Long J, Gilbert M. Molecular recognition at the protein-hydroxyapatite interface. Crit Rev Oral Biol Med 2003;14:370e6.

[35] Hay DI, Moreno EC. Statherin and the acidic proline-rich proteins. In: Tenovuo J, editor. human saliva: clinical chemistry and microbiology. Boca Raton, FL: CRC Press; 1989. pp. 131e50.

[36] García-Godoy F, Hicks M.J. Maintaining the integrity of the enamel surface: the role of dental biofilm, saliva and preventive agents in enamel demineraliza- tion and remineralization. J Am Dent Assoc 2008;139:25Se34S.

[37] Johnsson M, Levine M, Nancollas G. Hydroxyapatite binding domains in sali- vary proteins. Crit Rev Oral Biol Med 1993;4:371e8.

[38] Li Y, Chen X, Ribeiro A, Jensen E, Holmberg K, Rodriguez-Cabello J, et al. Hybrid nanotopographical surfaces obtained by biomimetic mineralization of statherininspired elastin-like recombinamers. Adv Healthc Mater 2014: 1e10. http://dx.doi.org/10.1002/adhm.201400015.

[39] Liao H, Andersson A-S, Sutherland D, Petronis S, Kasemo B, Thomsen P. Response of rat osteoblast-like cells to microstructured model surfaces in vitro. Biomaterials 2003;24:649e54.

[40] Yan H, Xufeng N, Wei S, Changdong G, Qingling F, Yubo F. Combined effects of mechanical strain and hydroxyapatite/collagen composite on osteogenic differentiation of rat bone marrow derived mesenchymal stem cells. J Nanomater 2013;2013.

[41] Dimitriou R, Tsiridis E, Giannoudis PV. Current concepts of molecular aspects of bone healing. Injury 2005;36:1392e 404.

[42] Sallach R, Cui W, Wen J, Martinez A, Conticello V, Chaikof E. Elastinmimetic protein polymers capable of physical and chemical crosslinking. Biomaterials 2009;30:409e22.

[43] Hrabchak C, Rouleau J, Moss I, Woodhouse K, Akens M, Bellingham C, et al. Assessment of biocompatibility and initial evaluation of genipin cross linked elastin-like polypeptides in the treatment of an osteochondral knee defect in rabbits. Acta Biomater 2010;6:2108e15.

[44] Kumar V, Caves J, Haller C, Dai E, Liu L, Grainger S, et al. Acellular vascular grafts generated from collagen and elastin analogs. Acta Biomater 2013;9: 8067e 74 .

[45] Kawasaki K. Weiss K. Mineralized tissue and vertebrate evolution: the secretory calcium-binding phosphoprotein gene cluster. Proc Natl Acad Sci U.S. A. 2003;100:4060e5. 